\title{
LRRK2 deficiency impacts ceramide metabolism in brain
}

Ruggero Ferrazza ${ }^{1},{\text { Susanna } \text { Cogo }^{2}, \text { Heather Melrose }^{3}, \text { Luigi Bubacco }^{2} \text {, Elisa Greggio }}^{2}$, Graziano Guella ${ }^{1,4}$, Laura Civiero $^{2 *}$, Nicoletta Plotegher ${ }^{2,5^{*}}$

${ }^{1}$ Bioorganic Chemistry Laboratory, Department of Physics, University of Trento, Via Sommarive 14, 38123 Povo (Trento), Italy

${ }^{2}$ Department of Biology, University of Padova, Via U. Bassi 58/b, 35131, Padova, Italy

${ }^{3}$ Department of Neuroscience, Mayo Clinic Jacksonville, Jacksonville, FL 32224, USA

${ }^{4}$ Biophysical Institute, CNR, Via alla Cascata 56/C, 38123, Povo (TN), Italy

${ }^{5}$ Present address: Department of Cell and Developmental Biology, University College London, Gower Street, WC1E 6BT London, UK

* To whom correspondence should be addressed: n.plotegher@ucl.ac.uk and laura.civiero@unipd.it 


\begin{abstract}
Mutations in $L R R K 2$ gene cause inherited Parkinson's disease (PD) and variations around LRRK2 act as risk factor for disease. Similar to sporadic disease, LRRK2-linked cases show late onset and, typically, the presence of proteinaceous inclusions named Lewy bodies (LBs) in neurons. Recently, defects on ceramide (Cer) metabolism have been recognized in PD. In particular, heterozygous mutations in the gene encoding for glucocerebrosidase (GBA1), a lysosomal enzyme converting glucosyl-ceramides (Glc-Cer) into Cer, increase the risk of developing PD. Although several studies have linked LRRK2 with membrane-related processes and autophagic-lysosomal pathway regulation, whether this protein impinges on the Cer pathway has not been addressed. Here, using a targeted lipidomics approach, we report an altered sphingolipid composition in $L r r k 2^{-/}$ mouse brains. In particular, we observe a significant increase of Cer levels in $\mathrm{Lrrk} 2^{-/}$mice and direct effects on GBA1. Collectively, our results suggest a link between LRRK2 and Cer metabolism, providing new insights into the possible role of this protein in sphingolipids metabolism, with implications for PD therapeutics.
\end{abstract}

Keywords: Lipidomics, sphingolipids, Parkinson's disease, glucocerebrosidase, Gaucher's disease, mass spectrometry. 


\section{INTRODUCTION}

Parkinson's disease (PD) is an incurable disorder of the nervous system that affects voluntary movements. It occurs as hereditary or sporadic condition that progressively impairs the functionality of dopaminergic neurons of the substantia nigra pars compacta and, at later stages, of other brain regions. Another pathological hallmark of PD is the presence of abnormal lipids and protein aggregates termed Lewy bodies (LBs) inside the nerve cells. The main constituent of these inclusions is an aggregated fibrillar form of $\alpha$ synuclein $(\mathrm{aS})[1]$.

Sphingolipids are particularly abundant in the nervous system and play important roles in cell function under physiological conditions and during disease development and progression. Several studies have provided evidence that sphingolipid levels are often altered in neurodegenerative diseases [2-4]. Ceramides (Cer), components of all major sphingolipid species in the brain, were found to have a defective homeostasis in neurodegenerative disorders with LBs pathology, including PD [2,5-7]. This class of lipids is generated by three pathways: $i$ ) the sphingomyelin hydrolysis that occurs at the plasma membrane, ii) the de novo synthesis taking place within endoplasmic reticulum (ER)/Golgi apparatus and iii) the salvage pathway in the endo-lysosomal compartment. Cer play a crucial role in the regulation of both intracellular and plasma membrane dynamics, and in the modulation of membrane protein activity and signaling [8]. Moreover, Cer modulate processes that involve intracellular organelles such as autophagy [9] or mitochondrial-mediated apoptosis [10]. Other lines of evidence link Cer balance to PD pathogenesis: first, mutations in GBA1 and SMPD1, encoding two enzymes that regulate the ceramide salvage pathway, cause inherited forms of lysosomal storage disorders (LSDs) characterized by LB inclusions and neurodegeneration $[11,12]$ and, second, common variants in these genes have been identified as risk factors for PD [13].

LRRK2 is mutated in families with autosomal dominantly inherited PD, and common variations around LRRK2 act as susceptibility factors for the disease [14,15]. LRRK2 is a large multi-domain protein exhibiting both GTPase and kinase activities, and the most common PD-linked G2019S mutation shows a three-fold increase in LRRK2 kinase activity both in vitro and in vivo [16,17]. G2019S carriers exhibit a phenotype nearly indistinguishable from idiopathic PD, with a late onset and often with LBs pathology [18]. Accumulating 
evidence indicates that LRRK2 is associated with membrane compartments $[19,20]$ where it phosphorylates key proteins involved in membrane remodeling $[17,21,22]$ and regulates different processes including autophagylysosome pathway [23], vesicular trafficking and protein sorting [14,24]. However, Lrrk $2^{-/}$mice or rats show a normal dopaminergic system, with subtle or no alterations in the number of dopaminergic neurons and in the levels of striatal dopamine [25].

Recently, a strong association among PD, the G2019S LRRK2 mutation and/or GBA1 mutations was documented [26]. Accordingly, altered GBA1 activity was measured in blood from LRRK2 G2019S carriers compared to non-carriers [27]. However, it is not known whether LRRK2 is directly or indirectly implicated in sphingolipid/ceramide metabolism and/or to GBA1 function. Here, we investigate whether LRRK2 impacts sphingolipid metabolism: using a targeted lipidomics approach, we look at the consequence of Lrrk2 knock-out on the sphingolipid composition in mouse brains. 


\section{MATERIALS AND METHODS}

\section{Animals}

C57BL/6 knock-out $\left(\right.$ Lrrk2 $\left.^{--}\right)$mice were provided by Dr. Heather Melrose. Housing and handling of mice were done in compliance with national guidelines. All animal procedures were approved by the Ethical Committee of the University of Padova and the Italian Ministry of Health (license 46/2012).

\section{Lipid extraction}

Brains were collected from three male $\mathrm{Lrrk}^{+/+}$and three male $\operatorname{Lrk} 2^{-/-}$mice at 1 year of age. Lipid extraction was performed through a protocol optimized for sphingolipids. Brains were homogenized in ultra-pure water and incubated on ice. $3 \mathrm{~mL}$ Folch solution (chloroform:methanol 2:1 v/v) were added to the homogenate and the solution was sonicated for $15 \mathrm{~min}$ at $4{ }^{\circ} \mathrm{C}$. Centrifugation at $16000 \mathrm{~g}$ for $15 \mathrm{~min}$ at $4{ }^{\circ} \mathrm{C}$ followed to recover the liquid phase [28]. The extracted samples were dried using a rotary evaporator, dissolved in $150 \mu \mathrm{L}$ HPLC-grade methanol (Sigma-Aldrich Co.) and stored in closed vials at $-20{ }^{\circ} \mathrm{C}$ until analysis.

\section{LC-MS analysis}

The LC-MS measurements were carried out using a Shimadzu High Performance LC system (CBM-20 A, equipped with the binary pump LC-20AB, Italy) working in reversed phase with a Kinetex C18 column (100 Å pore size, $4.6 \mathrm{~mm}$ ID, $2.6 \mu \mathrm{m}$ particle size, and $10 \mathrm{~cm}$ length, Phenomenex, Italy). The mobile phase was composed of solvent $\mathrm{A}$, methanol:water (7:3 v/v) with $12 \mathrm{mM}$ ammonium acetate, and solvent $\mathrm{B}$, methanol with $12 \mathrm{mM}$ ammonium acetate. The gradient elution program started with $70 \% \mathrm{~B}$, reached $100 \% \mathrm{~B}$ in 45 min, and was maintained at $100 \% \mathrm{~B}$ for $20 \mathrm{~min}$, at the steady flow rate of $1 \mathrm{~mL} / \mathrm{min}$.

The HPLC was combined with an Applied Biosystems API 3000 QQQ mass spectrometer equipped with an electrospray ion source. Each sample was injected twice (10 $\mu \mathrm{L}$ aliquots). Standard solutions were used to obtain the response factors needed to convert peak areas into molar quantities. The mass spectrometer was operated in Precursor Ion Scan Mode (PIS), in particular PIS of m/z 184 allowed to identify phosphatidylcholines (PC), lyso-phosphatidylcholines (Lyso-PC), plasmenyl-phosphatidylcholines (pPC) and sphingomyelins (SM), PIS of m/z 264 was used to characterize 18:1 ceramides (Cer 18:1), 18:1 glycosylceramides (Gly-Cer 18:1), 2-hydroxylated 18:1 glycosyl-ceramides (Gly-Cer 18:1(2-OH)) and sulfatides, 
whereas PIS of m/z 266 was employed for 18:0 ceramides (Cer 18:0) and 18:0 glycosyl-ceramides (Gly-Cer 18:0). Since the reversed-phase chromatography method we used did not allow to resolve the different glycosylated species, such as glucosyl-ceramides and galactosyl-ceramides [29], they are all referred to as glycosyl-ceramides (Gly-Cer).

\section{Lipidomics data analysis}

The LC-MS chromatograms were integrated using the proprietary software (Analyst 1.4.2), and the obtained molar amounts were analyzed using R environment.

To directly compare sphingolipid levels across samples, their molar amounts were normalized to the total lipid content, to obtain molar fractions. This approach allowed us to take into account the different biomasses of the samples. To compare changes in ceramides chain length and/or unsaturation across samples, the molar fraction of each ceramide was normalized to the total amount of ceramide d18 and expressed as percentage.

\section{Western blot}

Mouse brains were homogenized as previously described [30]. $20 \mu \mathrm{g}$ of protein samples were resolved on 4-20\% Tris-glycine polyacrylamide gels (Biorad) in SDS/Tris-glycine running buffer, transferred to polyvinylidenedifluoride (PVDF) membranes and probed with anti-LRRK2 MJFF2 (Epitomics Cat\# 3514-1, RRID:AB_10643781, 1:100), anti-GBA1 validated in gba KO mice model [31] (Cat\# AP1140, Calbiochem, 1:500) or anti-tubulin (Sigma, Cat\# T8328, RRID:AB_1844090, 1:5000) antibodies and then with horseradish peroxidase-conjugated anti-mouse IgG.

\section{GBA1 enzymatic activity}

The GBA1 enzymatic assay on brain lysates was performed as described elsewhere [31]. GBA1 activity was determined in samples (20 $\mu \mathrm{g}$ of total protein) by hydrolysis of $5 \mathrm{mM}$ 4-methylumbelliferyl-b-Dglucopyranoside (Cat\# M3633, Sigma) in McIIvaine buffer pH 5.4 in the presence of 22 mM sodium taurocholate at $37{ }^{\circ} \mathrm{C}$ for 1 hour. The reaction was stopped by adding $0.25 \mathrm{M}$ glycine $\mathrm{pH} 10.4$ and 4 methylumbelliferone and products quantified by fluorescence (Ls50, Perkin Elmer, Waltham, MA, USA). 10 $\mu$ M 
GBA1 inhibitor (CBE, Conduritol B Epoxide, Cat\# sc-201356, Santa Cruz) was added to the lysate as positive control. 


\section{RESULTS}

\section{8:1 Cer level is increased in Lrrk2 $^{-/-}$mouse brains}

In order to evaluate whether LRRK2 is involved in the regulation of the sphingolipid metabolism in the brain, we compared Cer, Gly-Cer, sulfatide and SM levels from brains of Lrrk2 ${ }^{-/}$mice to those in the control $\left(\operatorname{Lrrk}^{+/+}\right)$mice through a targeted LC-MS based approach ( $\mathrm{n}=3$ brains per condition analyzed separately). Data were normalized to the total lipids content to account for the different sample biomasses. The results are summarized in Figure 1, where the overall levels of identified sphingolipids in $L r r k 2^{-/}$mice (KO) are compared to those in the control $\mathrm{Lrrk}^{+/+}$(WT) mice. Statistical analysis (t-test) suggest that normalized Cer amount is significantly higher in $L r r k 2^{-/}$as compared to $L r r k 2^{+/+}$mice, suggesting that the absence of LRRK2 has an impact on Cer metabolism. In addition, the normalized amounts of SM, sulfatides and Gly-Cer show an interesting trend: SM and sulfatides tend to be higher in $\mathrm{Lrrk}^{-/}$samples, whereas Gly-Cer levels are lower. Interestingly, Cer, SM and sulfatides are all downstream of GBA1, while Gly-Cer, being partially constituted by Glc-Cer [29], are upstream. Since in our experimental setup we could not separate Glc-Cer and Gal-Cer, we verified if a measurable effect on the overall Gly-Cer level in neurons could be obtained by inhibiting GBA1 using CBE. The results show that when GBA1 activity is reduced by about $80 \%$ through $50 \mu \mathrm{M}$ CBE cronic treatment, a clear effect on the Gly-Cer level and on the Gly-Cer/Cer ratio can be appreciated in primary neurons (Supplementary Figure 1). Therefore, we calculated the overall molar ratio [Gly-Cer]/[Cer] on in $L r r k 2^{-/}$brains compared to controls. Figure 2 shows the comparison between samples and even though there is a clear trend, the difference is not significant. Taken together, these results point to an involvement of LRRK2 on Cer metabolism but further experiments are needed to verify a possible LRRK2-mediated dysregulation of GBA1.

\section{LRRK2 deficiency does not affect the Cer fatty acyl chain composition}

The relative amounts of different Cer species, as defined by their fatty acyl chain composition, regulate ceramide function in lipid membranes and signaling pathways and was found to be unbalanced in PD brain samples [5,32]. To assess whether the observed increase of total 18:1 Cer in brains was specific to certain acyl chains, we compared their relative amounts across samples, as described in the Methods. As shown in Figure 3, there are no significant differences between $L r r k 2^{+/+}$and $L r r k 2^{-/}$samples, and the intra-class profiles are 
indistinguishable. Collectively, our results suggest that whereas the total Cer amount is affected by Lrrk2 knockout, the intra-class Cer distribution is not perturbed.

\section{Lrrk $^{-/}$mouse brains show alterations in GBA1 enzymatic activity}

Considering GBA1 involvement in PD, we hypothesize that the detected imbalance in Cer levels might be due to (at least in part) a dysregulation of GBA1 activity in $L r r k 2^{-/}$brains. To further explore the impact of LRRK2 on the regulation of GBA1, we measured GBA1 protein levels in $\mathrm{Lrrk}^{+/+}$and $\mathrm{Lrrk}^{-/-}$brain lysates. Unexpectedly, we observed a significant downregulation of GBA1 in $L r r k 2^{-/}$brain lysates (Figure 4A-B; representative image of $n=3$ brains per condition; experiment repeated three times, 9 brains in total per condition, t-test). Next, to assess whether GBA1 activity is affected in $L r r k 2^{-/}$mice, we performed in vitro GBA1 activity assays on $\mathrm{Lrrk}^{+/+}$and $\mathrm{Lrrk}^{-/-}$brain lysates. To take into account the differences between total GBA1 amount in $\mathrm{Lrrk}^{+/+}$and $\mathrm{Lrrk}^{-/}$brain lysates, we calculated the specific GBA activity by normalizing the enzymatic activity (measured in lysates containing $20 \mu \mathrm{g}$ of total proteins) to the protein level (measured by western blot) per each genotype. The results show that GBA1 specific activity is increased in Lrrk2 $2^{-\%}$ brain lysates (Figure 4C, t-test), in agreement with the accumulation of Cer measured by the MS approach. 


\section{DISCUSSION}

In the present study we used a targeted lipidomics approach to identify possible sphingolipid alterations in brain samples of knock-out compared to wild-type Lrrk2 mice. To achieve this, we performed targeted LCMS measurements and focused on sphingolipids, whose alterations in brain tissue of PD patients have been previously reported [5] and have been associated with dysregulation in GBA1 level and activity [33].

We first observed a significant increase in the relative amount of Cer, together with a modest, nonsignificant increase in SM and sulfatides, and a modest, non-significant decrease in Gly-Cer. These variations suggest that LRRK2 deficiency affects sphingolipid metabolism, particularly Cer, which are the direct products of the reaction catalyzed by GBA1 in lysosomes. Specifically, the lack of LRRK2 is associated with a significant increase in GBA1 product, hinting that LRRK2 may regulate GBA1. However, by computing the Gly-Cer/Cer ratios we observed no significant differences, suggesting that the effect (if any) is order of magnitude lower compared to those exerted by CBE treatment in cortical neurons, or that the presence of Gal-Cer in the overall Gly-Cer may mask a measurable decrease in the Gly-Cer and therefore in Gly-Cer/Cer ratio [34]. To test the possibility that GBA1 is directly affected by the lack of LRRK2, we measured GBA1 protein amount and GBA1 enzymatic activity in brain lysates. Our results show that while GBA1 protein levels are reduced in $L r r k 2^{-/}$ brains as compared to wild-type, GBA1 specific activity is increased, a finding consistent with the accumulation of Cer observed with the lipidomics approach.

Our data, together with the reported increase in GBA1 activity in the blood samples from LRRK2 G2019S patients [27], further support the involvement of LRRK2 in GBA1 regulation and suggest that both the presence of LRRK2 and its kinase activity might be involved in Cer metabolism through GBA1 regulation. On the other hand, in idiopathic PD patients as well as in PD patients carrying GBA1 mutations and in GD patients, a decrease in GBA1 level is usually associated with a decrease in GBA1 activity [27,33,35-40] (Table 1). The overall picture that emerges from this analysis is that Cer metabolism is important in PD etiopathogenesis. The association of abnormal GBA1 activity with PD, higher in LRRK2 G2019S patients and in LRRK2 deficient systems and lower in both GBA1 mutation carriers as well as sporadic PD patients without GBA1 mutations, may reflect a distinct pathogenic mechanism for LRRK2-linked PD that deserves further investigation. 
Since LRRK2 functions as scaffold for several kinases and in the Wnt signalling by orchestrating their subcellular distribution and/or their interactions with upstream and downstream effectors [30,41], one possibility is that LRRK2 regulates GBA1 activity by affecting GBA1 localization, e.g. its delivery to lysosomes from the ER, which is controlled by lysosomal integral membrane protein type-2 (LIMP-2) [42]. Since an increase in GBA1 specific activity causes Cer accumulation, which we observed in $L r r k 2^{-/}$brains, we suggest that a feedback mechanism may occur, leading to a reduction in GBA1 expression level. A decreased GBA1 level may be reached through an increase in its degradation rate or through the downregulation of GBA1 gene. However, it is worth noting that also the other pathways involved in Cer regulation described in the introduction may be affected directly or indirectly by LRRK2 depletion.

To conclude, we propose that LRRK2 may play an important role in the sphingolipid metabolism, by affecting GBA1 regulation. Future studies are needed to confirm this link using independent models and to gain mechanistic insights into how LRRK2 regulates GBA1 function. Moreover, more needs to be done to understand if LRRK2 directly or indirectly regulates Cer metabolism through other pathways. Our results together with other recently published papers $[2,5,7,11,27,33]$ suggest that PD shares several features with sphingolipid disorders, opening new avenues for the identification of novel therapeutic strategies.

\section{Acknowledgments:}

This work was supported by the Telethon - Italy [Grant no. GGP12237]; Michael J. Fox Foundation and Unipd [Progetto di Ateneo - 2014]; Centre for Integrative Biology (CIBIO) - Italy (RF); Marie Skłodowska-Curie Individual Fellowship (NP); Unipd [Assegno Senior - 2014] (LC). 


\section{REFERENCES}

[1] K.A. Jellinger, Neuropathology of sporadic Parkinson's disease: evaluation and changes of concepts., Mov. Disord. 27 (2012) 8-30. doi:10.1002/mds.23795.

[2] J. Bras, A. Singleton, M.R. Cookson, J. Hardy, Emerging pathways in genetic Parkinson's disease: Potential role of ceramide metabolism in Lewy body disease., FEBS J. 275 (2008) 5767-73. doi:10.1111/j.1742-4658.2008.06709.x.

[3] N.J. Haughey, Sphingolipids in neurodegeneration., Neuromolecular Med. 12 (2010) 301-5. doi:10.1007/s12017-010-8135-5.

[4] N. Fabelo, V. Martín, G. Santpere, R. Marín, L. Torrent, I. Ferrer, M. Díaz, Severe alterations in lipid composition of frontal cortex lipid rafts from Parkinson's disease and incidental Parkinson's disease., Mol. Med. 17 (2011) 1107-18. doi:10.2119/molmed.2011.00119.

[5] S.K. Abbott, H. Li, S.S. Muñoz, B. Knoch, M. Batterham, K.E. Murphy, G.M. Halliday, B. Garner, Altered ceramide acyl chain length and ceramide synthase gene expression in $\begin{array}{llllll}\text { Parkinson's } & \text { disease., } & \text { Mov. } & \text { Disord. } & 29 & \text { (2014) }\end{array}$ http://www.ncbi.nlm.nih.gov/pubmed/24822250.

[6] A.S. Don, J.-H.T. Hsiao, J.M. Bleasel, T.A. Couttas, G.M. Halliday, W.S. Kim, Altered lipid levels provide evidence for myelin dysfunction in multiple system atrophy., Acta Neuropathol. Commun. 2 (2014) 150. doi:10.1186/s40478-014-0150-6.

[7] M.M. Mielke, W. Maetzler, N.J. Haughey, V.V.R. Bandaru, R. Savica, C. Deuschle, T. Gasser, A.-K. Hauser, S. Gräber-Sultan, E. Schleicher, D. Berg, I. Liepelt-Scarfone, Plasma ceramide and glucosylceramide metabolism is altered in sporadic Parkinson's disease and associated with cognitive impairment: a pilot study., PLoS One. 8 (2013) e73094. doi:10.1371/journal.pone.0073094.

[8] E. Posse de Chaves, S. Sipione, Sphingolipids and gangliosides of the nervous system in membrane function and dysfunction., FEBS Lett. $584 \quad$ (2010) 1748-59. doi:10.1016/j.febslet.2009.12.010.

[9] E.B. Harvald, A.S.B. Olsen, N.J. Færgeman, Autophagy in the light of sphingolipid metabolism., Apoptosis. 20 (2015) 658-70. doi:10.1007/s10495-015-1108-2.

[10] A. Kogot-Levin, A. Saada, Ceramide and the mitochondrial respiratory chain, Biochimie. 100 (2014) 88-94. doi:10.1016/j.biochi.2013.07.027.

[11] G. Stern, Niemann-Pick's and Gaucher's diseases., Parkinsonism Relat. Disord. 20 Suppl 1 (2014) S143-6. doi:10.1016/S1353-8020(13)70034-8.

[12] J.-N. Foo, H. Liany, J.-X. Bei, X.-Q. Yu, J. Liu, W.-L. Au, K.M. Prakash, L.C. Tan, E.-K. Tan, Rare lysosomal enzyme gene SMPD1 variant (p.R591C) associates with Parkinson's disease., Neurobiol. Aging. 34 (2013) 2890.e13-5. doi:10.1016/j.neurobiolaging.2013.06.010.

[13] Z. Gan-Or, L.J. Ozelius, A. Bar-Shira, R. Saunders-Pullman, A. Mirelman, R. Kornreich, M. Gana-Weisz, D. Raymond, L. Rozenkrantz, A. Deik, T. Gurevich, S.J. Gross, N. SchreiberAgus, N. Giladi, S.B. Bressman, A. Orr-Urtreger, The p.L302P mutation in the lysosomal enzyme gene SMPD1 is a risk factor for Parkinson disease., Neurology. 80 (2013) 1606-10. doi:10.1212/WNL.0b013e31828f180e.

[14] I.F. Mata, W.J. Wedemeyer, M.J. Farrer, J.P. Taylor, K.A. Gallo, LRRK2 in Parkinson's disease: protein domains and functional insights., Trends Neurosci. 29 (2006) 286-93. 
doi:10.1016/j.tins.2006.03.006.

[15] S. Lesage, A. Brice, Parkinson's disease: from monogenic forms to genetic susceptibility factors, Hum. Mol. Genet. 18 (2009) R48-R59. doi:10.1093/hmg/ddp012.

[16] E. Greggio, I. Zambrano, A. Kaganovich, A. Beilina, J.-M. Taymans, V. Daniëls, P. Lewis, S. Jain, J. Ding, A. Syed, K.J. Thomas, V. Baekelandt, M.R. Cookson, The Parkinson diseaseassociated leucine-rich repeat kinase 2 (LRRK2) is a dimer that undergoes intramolecular autophosphorylation., J. Biol. Chem. 283 (2008) 16906-14. doi:10.1074/jbc.M708718200.

[17] M. Steger, F. Tonelli, G. Ito, P. Davies, M. Trost, M. Vetter, S. Wachter, E. Lorentzen, G. Duddy, S. Wilson, M.A. Baptista, B.K. Fiske, M.J. Fell, J.A. Morrow, A.D. Reith, D.R. Alessi, M. Mann, Phosphoproteomics reveals that Parkinson's disease kinase LRRK2 regulates a subset of Rab GTPases, Elife. 5 (2016). doi:10.7554/eLife.12813.

[18] H.F. Chien, T.R. Figueiredo, M.A. Hollaender, F. Tofoli, L.T. Takada, L. da V. Pereira, E.R. Barbosa, Frequency of the LRRK2 G2019S mutation in late-onset sporadic patients with Parkinson's disease., Arq. Neuropsiquiatr. $72 \quad$ (2014) 356-9. http://www.ncbi.nlm.nih.gov/pubmed/24863511.

[19] J. Schapansky, J.D. Nardozzi, F. Felizia, M.J. LaVoie, Membrane recruitment of endogenous LRRK2 precedes its potent regulation of autophagy., Hum. Mol. Genet. 23 (2014) 4201-14. doi:10.1093/hmg/ddu138.

[20] N.G. James, M.A. Digman, E. Gratton, B. Barylko, X. Ding, J.P. Albanesi, M.S. Goldberg, D.M. Jameson, Number and brightness analysis of LRRK2 oligomerization in live cells., Biophys. J. 102 (2012) L41-3. doi:10.1016/j.bpj.2012.04.046.

[21] S. Matta, K. Van Kolen, R. da Cunha, G. van den Bogaart, W. Mandemakers, K. Miskiewicz, P.J. De Bock, V.A. Morais, S. Vilain, D. Haddad, L. Delbroek, J. Swerts, L. Chávez-Gutiérrez, G. Esposito, G. Daneels, E. Karran, M. Holt, K. Gevaert, D.W. Moechars, B. De Strooper, P. Verstreken, LRRK2 controls an EndoA phosphorylation cycle in synaptic endocytosis., Neuron. 75 (2012) 1008-21. doi:10.1016/j.neuron.2012.08.022.

[22] E. Belluzzi, A. Gonnelli, M.-D. Cirnaru, A. Marte, N. Plotegher, I. Russo, L. Civiero, S. Cogo, M.P. Carrion, C. Franchin, G. Arrigoni, M. Beltramini, L. Bubacco, F. Onofri, G. Piccoli, E. Greggio, LRRK2 phosphorylates pre-synaptic N-ethylmaleimide sensitive fusion (NSF) protein enhancing its ATPase activity and SNARE complex disassembling rate., Mol. Neurodegener. 11 (2016) 1. doi:10.1186/s13024-015-0066-z.

[23] S.J. Orenstein, S.-H. Kuo, I. Tasset, E. Arias, H. Koga, I. Fernandez-Carasa, E. Cortes, L.S. Honig, W. Dauer, A. Consiglio, A. Raya, D. Sulzer, A.M. Cuervo, Interplay of LRRK2 with chaperone-mediated autophagy, Nat. Neurosci. 16 (2013) 394-406. doi:10.1038/nn.3350.

[24] G. Piccoli, S.B. Condliffe, M. Bauer, F. Giesert, K. Boldt, S. De Astis, A. Meixner, H. Sarioglu, D.M. Vogt-Weisenhorn, W. Wurst, C.J. Gloeckner, M. Matteoli, C. Sala, M. Ueffing, LRRK2 controls synaptic vesicle storage and mobilization within the recycling pool., J. Neurosci. 31 (2011) 2225-37. doi:10.1523/JNEUROSCI.3730-10.2011.

[25] K.M. Hinkle, M. Yue, B. Behrouz, J.C. Dächsel, S.J. Lincoln, E.E. Bowles, J.E. Beevers, B. Dugger, B. Winner, I. Prots, C.B. Kent, K. Nishioka, W.-L. Lin, D.W. Dickson, C.J. Janus, M.J. Farrer, H.L. Melrose, LRRK2 knockout mice have an intact dopaminergic system but display alterations in exploratory and motor co-ordination behaviors., Mol. Neurodegener. 7 (2012) 25. doi:10.1186/1750-1326-7-25. 
[26] M. Spitz, J.S. Pereira, D.H. Nicareta, G. de M. Abreu, E.F. Bastos, T.L. Seixas, M.M.G. Pimentel, Association of LRRK2 and GBA mutations in a Brazilian family with Parkinson's disease., Parkinsonism Relat. Disord. 21 (2015) 825-6. doi:10.1016/j.parkreldis.2015.03.029.

[27] R.N. Alcalay, O.A. Levy, C.H. Waters, S. Fahn, B. Ford, S.-H. Kuo, P. Mazzoni, M.W. Pauciulo, W.C. Nichols, Z. Gan-Or, G.A. Rouleau, W.K. Chung, P. Wolf, P. Oliva, J. Keutzer, K. Marder, X. Zhang, Glucocerebrosidase activity in Parkinson's disease with and without GBA mutations, Brain. 138 (2015) 2648-2658. doi:10.1093/brain/awv179.

[28] Y. Yu, L. Vidalino, A. Anesi, P. Macchi, G. Guella, A lipidomics investigation of the induced hypoxia stress on HeLa cells by using MS and NMR techniques., Mol. Biosyst. 10 (2014) 87890. doi:10.1039/c3mb70540d.

[29] R.L. Shaner, J.C. Allegood, H. Park, E. Wang, S. Kelly, C.A. Haynes, M.C. Sullards, A.H. Merrill, Quantitative analysis of sphingolipids for lipidomics using triple quadrupole and quadrupole linear ion trap mass spectrometers., J. Lipid Res. 50 (2009) 1692-707. doi:10.1194/jlr.D800051-JLR200.

[30] L. Civiero, M.D. Cirnaru, A. Beilina, U. Rodella, I. Russo, E. Belluzzi, E. Lobbestael, L. Reyniers, G. Hondhamuni, P.A. Lewis, C. Van den Haute, V. Baekelandt, R. Bandopadhyay, L. Bubacco, G. Piccoli, M.R. Cookson, J.-M. Taymans, E. Greggio, Leucine-rich repeat kinase 2 interacts with p21-activated kinase 6 to control neurite complexity in mammalian brain., J. Neurochem. 135 (2015) 1242-56. doi:10.1111/jnc.13369.

[31] L.D. Osellame, A.A. Rahim, I.P. Hargreaves, M.E. Gegg, A. Richard-Londt, S. Brandner, S.N. Waddington, A.H. V Schapira, M.R. Duchen, Mitochondria and quality control defects in a mouse model of Gaucher disease--links to Parkinson's disease., Cell Metab. 17 (2013) 941-53. doi:10.1016/j.cmet.2013.04.014.

[32] O. Ben-David, A.H. Futerman, The role of the ceramide acyl chain length in neurodegeneration: involvement of ceramide synthases., Neuromolecular Med. 12 (2010) 341-50. doi:10.1007/s12017-010-8114-x.

[33] K.E. Murphy, A.M. Gysbers, S.K. Abbott, N. Tayebi, W.S. Kim, E. Sidransky, A. Cooper, B. Garner, G.M. Halliday, Reduced glucocerebrosidase is associated with increased -synuclein in sporadic Parkinson's disease, Brain. 137 (2014) 834-848. doi:10.1093/brain/awt367.

[34] M. Boutin, Y. Sun, J.J. Shacka, C. Auray-Blais, Tandem Mass Spectrometry Multiplex Analysis of Glucosylceramide and Galactosylceramide Isoforms in Brain Tissues at Different Stages of Parkinson Disease, Anal. Chem. 88 (2016) 1856-1863. doi:10.1021/acs.analchem.5b04227.

[35] D.C. Schöndorf, M. Aureli, F.E. McAllister, C.J. Hindley, F. Mayer, B. Schmid, S.P. Sardi, M. Valsecchi, S. Hoffmann, L.K. Schwarz, U. Hedrich, D. Berg, L.S. Shihabuddin, J. Hu, J. Pruszak, S.P. Gygi, S. Sonnino, T. Gasser, M. Deleidi, iPSC-derived neurons from GBA1associated Parkinson's disease patients show autophagic defects and impaired calcium homeostasis., Nat. Commun. 5 (2014) 4028. doi:10.1038/ncomms5028.

[36] M.E. Gegg, D. Burke, S.J.R. Heales, J.M. Cooper, J. Hardy, N.W. Wood, A.H. V Schapira, Glucocerebrosidase deficiency in substantia nigra of parkinson disease brains, Ann. Neurol. 72 (2012) 455-463. doi:10.1002/ana.23614.

[37] H.-J. Kim, B. Jeon, J. Song, W.-W. Lee, H. Park, C.-W. Shin, Leukocyte glucocerebrosidase and $\beta$-hexosaminidase activity in sporadic and genetic Parkinson disease., Parkinsonism Relat. Disord. 23 (2016) 99-101. doi:10.1016/j.parkreldis.2015.12.002. 
[38] D. Chiasserini, S. Paciotti, P. Eusebi, E. Persichetti, A. Tasegian, M. Kurzawa-Akanbi, P.F. Chinnery, C.M. Morris, P. Calabresi, L. Parnetti, T. Beccari, Selective loss of glucocerebrosidase activity in sporadic Parkinson's disease and dementia with Lewy bodies, Mol. Neurodegener. 10 (2015) 15. doi:10.1186/s13024-015-0010-2.

[39] T. Farfel-Becker, E.B. Vitner, S.L. Kelly, J.R. Bame, J. Duan, V. Shinder, A.H. Merrill, K. Dobrenis, A.H. Futerman, Neuronal accumulation of glucosylceramide in a mouse model of neuronopathic Gaucher disease leads to neurodegeneration, Hum. Mol. Genet. 23 (2014) 843854. doi:10.1093/hmg/ddt468.

[40] M.E. Gegg, L. Sweet, B.H. Wang, L.S. Shihabuddin, S.P. Sardi, A.H.V. Schapira, No evidence for substrate accumulation in Parkinson brains with GBA mutations, Mov. Disord. 30 (2015) 1085-1089. doi:10.1002/mds.26278.

[41] D.C. Berwick, K. Harvey, LRRK2 functions as a Wnt signaling scaffold, bridging cytosolic proteins and membrane-localized LRP6., Hum. Mol. Genet. 21 (2012) 4966-79. doi:10.1093/hmg/dds342.

[42] D. Reczek, M. Schwake, J. Schröder, H. Hughes, J. Blanz, X. Jin, W. Brondyk, S. Van Patten, T. Edmunds, P. Saftig, LIMP-2 is a receptor for lysosomal mannose-6-phosphate-independent targeting of beta-glucocerebrosidase., Cell. 131 (2007) 770-83. doi:10.1016/j.cell.2007.10.018. 


\section{FIGURE LEGENDS}

Figure 1: Comparisons of sphingolipid classes between $\mathrm{Lrrk2}^{+/+}$and $\mathrm{Lrrk}^{-/}$mouse brain extracted lipids. Sphingolipids were measured by LC-MS in $\operatorname{Lrrk}^{+/+}(\mathrm{WT})$ and $L r r k 2^{-/-}(\mathrm{KO})$ mouse brains and Cer 18:1 levels were significantly increased in $L r r k 2^{-/}$brain samples, compared to controls. Relative levels are expressed as molar fractions (normalized to the total lipid content in the sample).

Figure 2: Gly-Cer/Cer ratio is comparable in $\mathrm{Lrrk}^{+/+}$and $\mathrm{Lrrk}^{-/-}$mouse brains.

Gly-Cer/Cer ratio is slightly lower in $\operatorname{Lrrk}^{-/}(\mathrm{KO})$ mouse brains, hinting to a possible dysfunction in GBA1, the key enzyme converting Glc-Cer to Cer. However, the difference is not significant as measured by t-test $(\mathrm{p}=0.14)$ and may be solely due to the significant difference observed in Cer levels.

Figure 3: Intra-class distribution of Cer 18:1 in $\mathrm{Lrrk}^{+/+}$and $\mathrm{Lrrk2}^{-/-}$mouse brain extracted lipids.

The Cer 18:1 intra-class distributions are indistinguishable between $L r r k 2^{+/+}(\mathrm{WT})$ and $L r r k 2^{-/}$(KO) samples, meaning that the increase in Cer levels following the knocking-out of Lrrk2 is not specific for Cer with certain acyl chains but it equally affects them.

Figure 4: GBA1 protein is downregulated and GBA1 specific activity is higher in $\mathrm{Lrrk}^{-/-}$mouse brains. A. Western blot of different brain lysates for GBA1 protein level (and the relative LRRK2 level) for Lrrk2 $^{+/+}$ and $\mathrm{Lrrk}^{-/}$mice and (B) the relative quantification ( $\mathrm{n}=9$ for each genotype). The result shows that there is a significant reduction in GBA1 protein levels in the knock-out mice for LRRK2, suggesting that the observed increase in Cer is due to other mechanisms affected by LRRK2 depletion.

C. GBA1 specific activity was calculated normalizing the GBA1 activity measured for the lysate of each genotype $(20 \mu \mathrm{g}$ total protein) by the GBA1 expression level as measured by western blot ( $\mathrm{n}=9$ for each genotype). Asterisks indicate statistical significance by t-test $(* \mathrm{p}<0.05)$.

Table 1. Summary of GBA1 activity/level and Cer/Gly-Cer amount in different PD and GD models. 


\begin{tabular}{|c|c|c|c|}
\hline Idiopathic PD patients & $\begin{array}{c}\text { GBA activity } \\
\downarrow(33,36,38)\end{array}$ & GBA level & GBA specific activity \\
\hline $\begin{array}{c}\text { PD patients carrying GBA } \\
\text { mutations }\end{array}$ & $\begin{array}{c}\text { Blood } \downarrow(27) \quad \text { Brain } \\
\downarrow(36)\end{array}$ & Brain $\downarrow(36)$ & Brain (probably =) \\
\hline GD patients or gba-/- mice & $\begin{array}{c}\text { Blood } \downarrow(27) \quad \text { Brain } \\
\downarrow(39)\end{array}$ & Brain $\downarrow(39)$ & Brain (probably =) \\
\hline LRRK2-linked PD patients & Blood $\uparrow(27)$ & & Brain $\uparrow$ \\
\hline Lrrk2-/- mice & Brain $=$ & Brain $\downarrow$ & \\
\hline
\end{tabular}



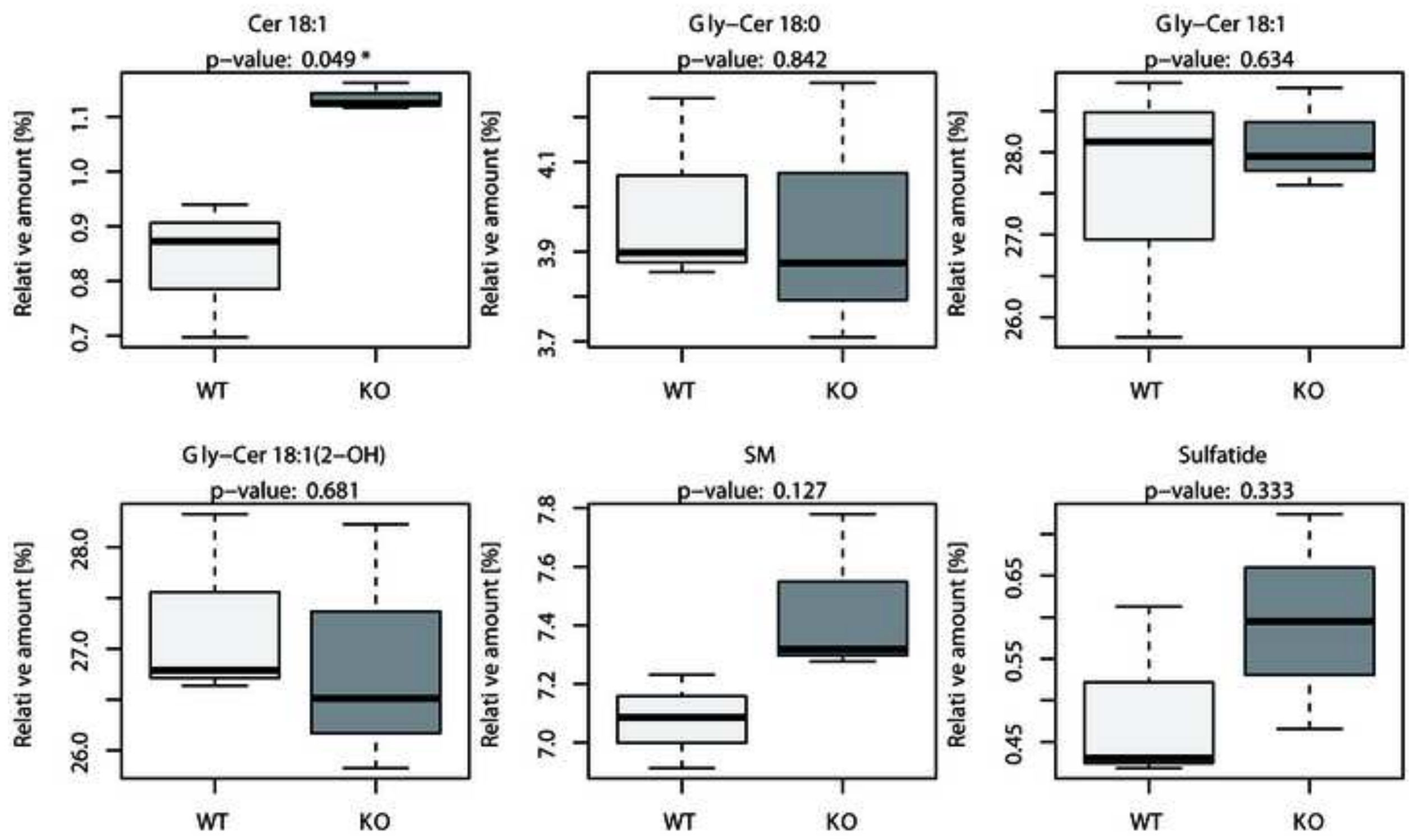
Figure 2
Click here to download high resolution image

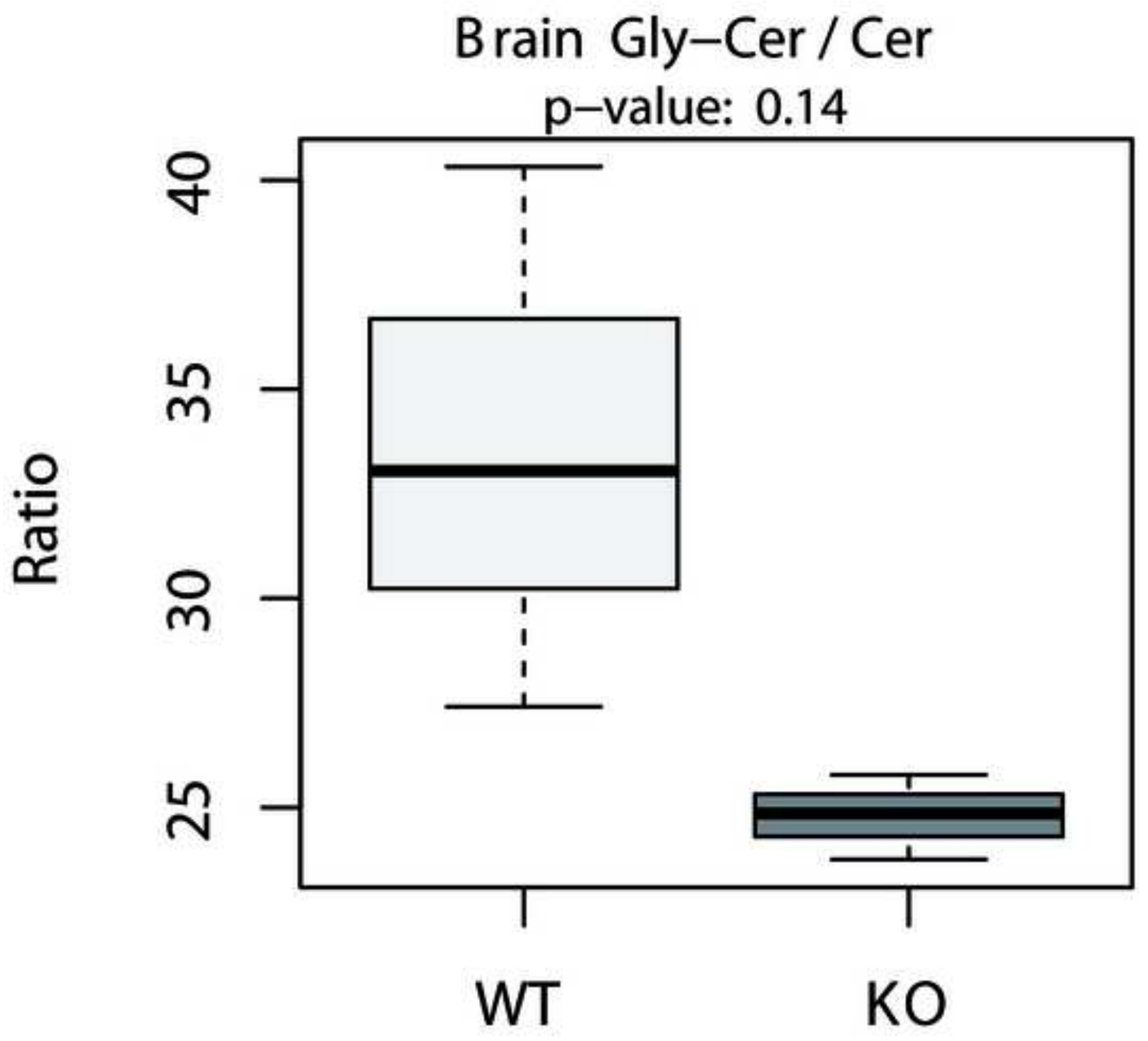




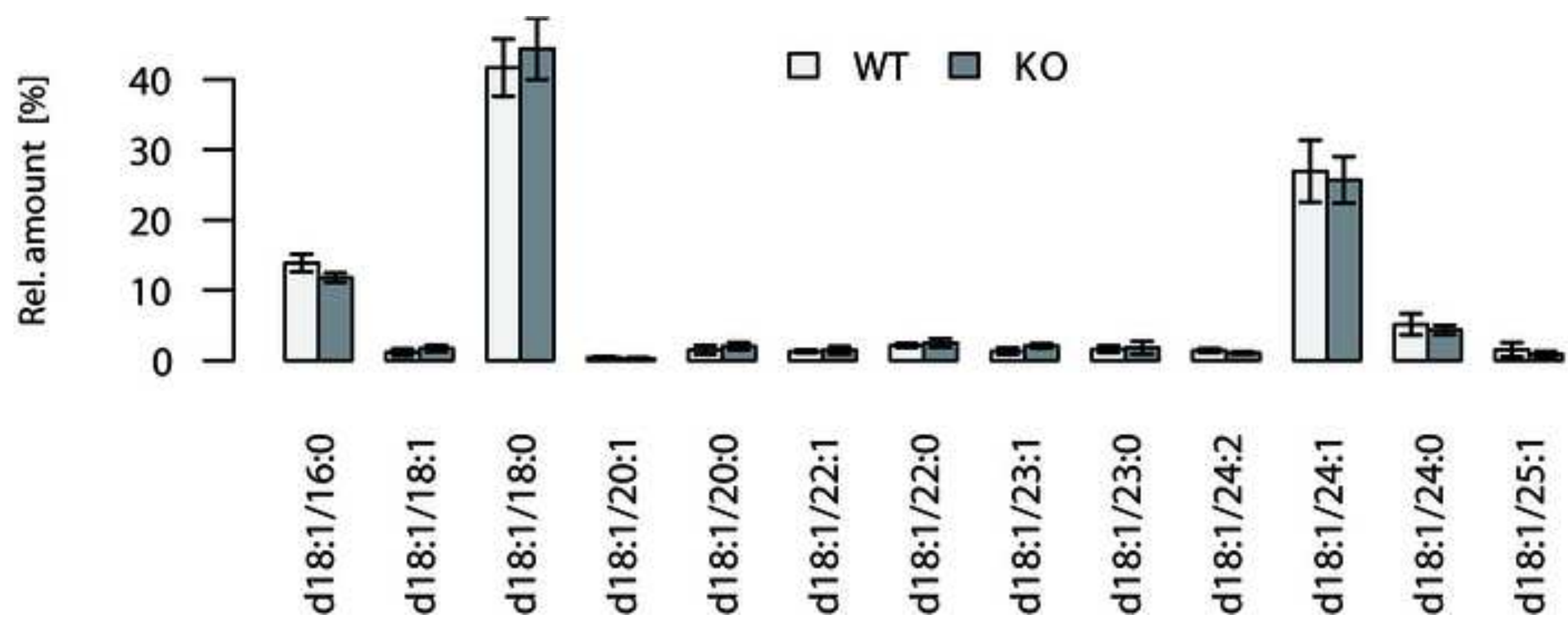


A

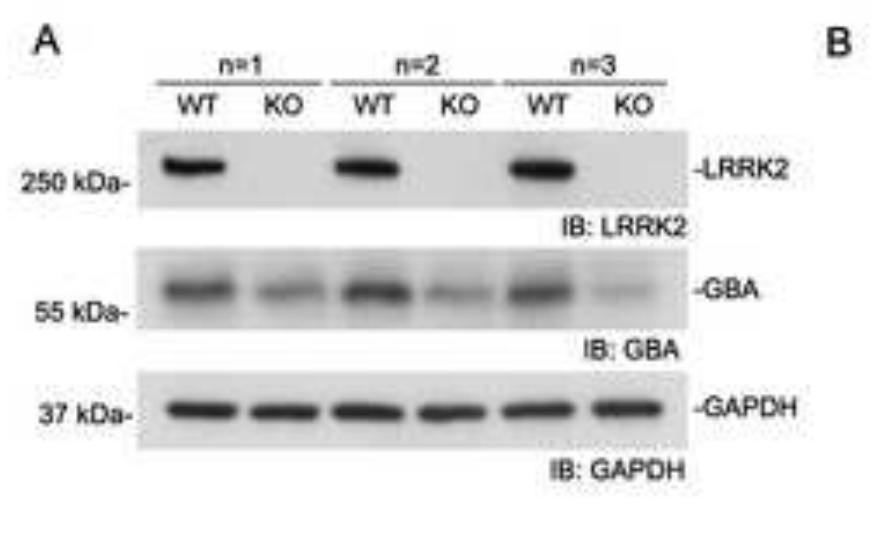

IB: GAPDH
Figure 4
Click here to download high resolution image
C

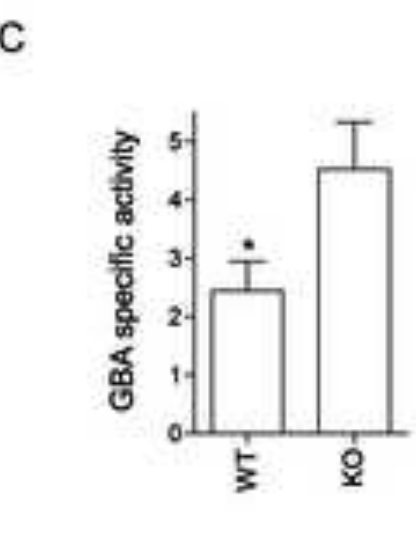

$$
\text { 胥 }
$$

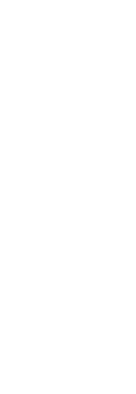

西
B

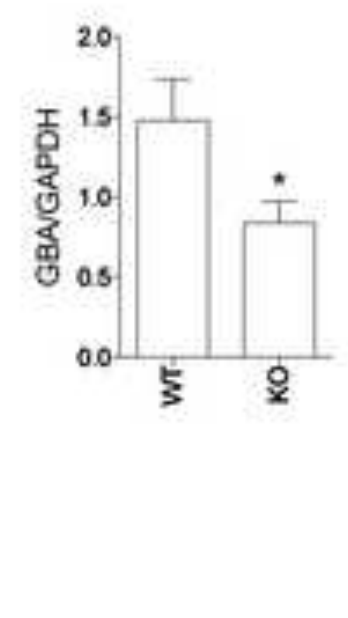

C

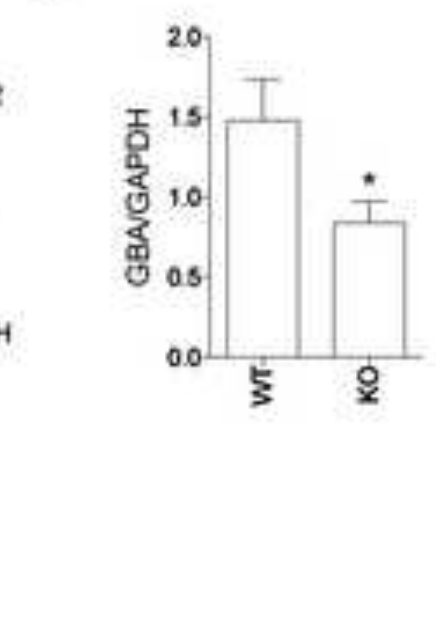

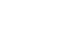

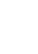


Electronic Supplementary Material (online publication only)
Click here to download Electronic Supplementary Material (c

Click here to download Electronic Supplementary Material (online publication only): 03-08-2016_Supplementary.docx 